\title{
Effect of Antenatal Pelvic Floor Muscle Exercise on Postnatal Stress Urinary Incontinence in Primigravida - An Comparative Experimental Study
}

\author{
Rejisha TR* \\ Department of Physiotherapy, Sanjay Gandhi Institute of Orthopedics and Trauma, Bangalore, India \\ *Corresponding author: Rejisha TR, Department of Physiotherapy, College of Physiotherapy, Sanjay Gandhi Institute of Orthopedics and Trauma, \\ Bangalore, India
}

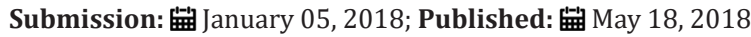

\section{Introduction}

Women health and physiotherapy in gynaecology have been a matter of concern since 1950's, but due to social and cultural limitations the problems of womanhood have been under reported. "A woman's health is her total well-being, not determined solely by biological factors and reproduction, but also by effects of work load, nutrition, stress, war and migration, among others" [1]. Basic health care, family planning and obstetric services are essential for women - yet they remain unavailable to millions. Genderequitable approaches to health are needed to enable women's full participation in the planning and delivery of health services. Some of the major health and mental challenges that women face today include pregnancy and its complications, AIDS, cervical cancer, breast cancer, depression, anxiety, etc.

Postpartum Stress Urinary Incontinence is one such socially and emotionally challenging problem faced by women with major economical and psychological effects on society [2]. Urinary incontinence (UI), defined by the International Continence Society as "the complaint of any involuntary leakage of urine is a common and distressing medical condition, severely affecting quality of life (QOL) [3]. The problem leads to many women adopting detrimental changes in lifestyle to combat it and may even lead to feelings of shame and depression or even avoidance of social, work events, and sexual activities3. There is a significant impact of urinary incontinence on quality of life. Mitigating the effect of urinary incontinence and improving their quality of life in women will require further understanding of their coping skills and their perceptions of themselves [4]. Co morbid conditions such as urinary tract infections, skin problems such as rashes, infections, and sores occur due to constantly wet skin. Economic burden due to increased costs and efforts for linen washing and healthcare adds to the consequences of this condition [3].

Studies confirm the considerable impact of urinary incontinence on HRQoL in a treatment seeking population, as demonstrated by the disease-specific quality of life scale and by the high percentage of patients who were bothered by their symptoms [5]. The epidemiology of stress urinary incontinence is variable according to the country being studied. Some estimates suggests that only $10-20 \%$ of patients will seek medical care for their pelvic floor dysfunction due to embarrassment, lack of knowledge about all the treatment options available and the thought that nothing can be done as this is just a normal part of motherhood [6]. This contributes to some difficulties with the epidemiology. Some studies have reported up to $77 \%$ women having some degree of SUI6 Prevalence of stress incontinence in pregnancy is 10-67\% [79] and in postpartum it is $29-31 \%$ [7]. Its incidence is estimated to be $40-82 \%$ [10].

Most of the studies from developed and developing countries confirm that the prevalence of urinary incontinence substantially increases during pregnancy [11]. In India there is very scarce data available regarding urinary incontinence and other urological problems during pregnancy. The prevalence of urinary incontinence and urinary problems was much higher during the third trimester of pregnancy than in second or first trimesters.

There are many factors affecting the continence of women. Some of them are age, parity, mode of delivery [12], BMI, obesity, surgery, constipation, and chronic respiratory problems $[13,14]$. Prior vaginal delivery is also a risk factor with prevalence of urinary incontinence being $42.81 \%$ in women who had prior vaginal deliveries as compared to $27.27 \%$ in women who had no vaginal delivery [12]. There is a common notion that urinary incontinence is less prevalent in India and other developing countries. Almost 1 in 10 women reported suffering from episodes of postnatal SUI in India $[8,15]$. Yet postnatal stress urinary incontinence remains a major issue among the young female population of India. This is probably attributable to the lack of awareness amongst women who consider it to be a "normal" physiological process during pregnancy and are hesitant to report their problems unless specifically asked, or unless they perceive a significant deterioration in quality-of-life. 
The need to ask patients direct questions about urological symptoms during and after pregnancy to identify the exact nature of these conditions and to offer appropriate treatment for their symptoms so they do not suffer in silence, it is important to get the proper data regarding the problem so as to provide the exact picture of the scenario [16].

\section{Objectives of the Study}

The objective of this study was to analyse the effect of antenatal pelvic floor muscle training on postnatal SUI in primigravida, and to compare the effect of antenatal pelvic floor muscle exercise on postnatal SUI in terms of incidence and severity in groups with and without pelvic floor muscle training undergoing a normal delivery.

\section{Procedure}

60 Subjects, ranging from an age of 24 to 30 in the 6th month of the pregnancy who were primigravid as with single foetus and had no high risk diagnosed in the course of their pregnancy were identified from different social and economical settings in different women health centres in Bangalore. Subjects with known case of SUI, multiple pregnancies, multiple foetuses and beyond the age limit were excluded from the study.

Subjects, who satisfied the selection criteria, were explained about the physiological changes during antenatal period, perinatal period and postnatal period. Once it was the understood by the subjects, they were allocated to experimental and control group by convenient sampling technique. Respecting the subject's confidentiality and privacy the informed consent was obtained from the subjects.

The experimental group (group GE): Subjects who were allocated into the experimental group were given pelvic floor muscle training from their 6th month of pregnancy. The correct identification of the pelvic floor muscle had to be taught to the subjects for correctly exercising the target muscles and thus obtaining a better outcome.

Once the subject was confident about performing the exercise she was asked to repeat the exercise as follows:

For Slow contracting fibbers of pelvic floor muscles Slow Kegels exercise 17 was taught to the subjects. Here the subjects were trained to contract their pelvic muscles for 10 seconds and then relax them for 10 seconds. Along with the breathing pattern ie breathe in during relaxation period and breathe out during contraction. The subjects were asked to repeat the Kegel exercises 8 to 12 times each session, and do at least 3 sessions a day.

For fast contracting fibers of pelvic floor muscles fast Kegels exercise was taught to the subjects [17]. The subjects were asked to rapidly contract the PFMs, 5-10 contraction at a stretch and repeated 3 times daily 17 . As the subjects progressed through their pregnancy they were asked to change the position from lying to sitting to standing. As the gravity and the weight of the uterus is eliminated in lying position, supporting in sitting and resistive in standing position, the PFMT can be done according to the overloading principle of muscle training. Thus the resistance to PFM is at the lowest in lying position, assisted or moderate in the sitting position and high in the standing position. Thus the exercise can be done in progression [17]. The subjects were seen once a week for supervised exercise sessions, but was instructed to do the exercise 3-4 times a week for 15 to 20 weeks (till term) [18].

\section{The control group (GC)}

were given standard information about the antenatal and postnatal changes and breathing exercise, but not the Kegels exercise from the 6th month of pregnancy, hence they were in an inactive control treatment.

Those subjects among the experimental and the GC who underwent a normal vaginal delivery not a c-section or other assisted deliveries like the forceps delivery or the vacuum delivery were interviewed at the end of their 1 st postnatal month in regard to the SUI experienced by them using leakage index 19 as the outcome measure.

There were 60 selected subjects with 30 each in experimental and the GC. The drop outs of the study was 08 from the experimental group and 0 from the GC, the total no of subjects who underwent normal delivery in experimental group was 13 and in the GC 17. Subjects who underwent c-sessions was 3 in the experimental group and 5 in the GC, and those who underwent assisted delivery was 6 in experimental group and 8 in GC.

Outcome measure: Leakage index 20 this index contains thirteen types of physical exertions which trigger urinary leakage in women with SUI: laughing, coughing, sneezing, rising from lying to a sitting position, walking on the level, walking uphill, walking downhill, jumping with legs together, jumping with legs in subsequent abduction- and adduction, running, lifting, and sudden and rapid movements. The subjects who underwent a normal delivery in both the experimental group and the GC were asked to do these activities form the 14th day of delivery till the completion of the 1st postpartum month. Then the subjects were interviewed, the subjects were asked to record the degree of SUI for each parameter on a five point scale $(1=$ never, $2=$ seldom, $3=$ sometimes, $4=$ often, $5=$ =always). The mean was calculated as an overall index." The interview was repeated after 2 weeks also. And the average score was calculated.

\section{Result}

When comparing the post delivery leakage index in the experimental group and the control group, with values in the experimental group to be 1.0-2.61 and control group to be 1.0-3.73 with mean standard deviation of $1.48 \pm 0.49$ in experimental group and $2.50 \pm 1.16$ in the control group with $95 \%$ CI to be $1.18-1.79$ and 1.90-3.09 respectively, the $\mathrm{P}$ value was found to be $\mathrm{P}=0.006$ and the effect size was found to be 1.06(L). Hence it is statistically significant; therefore the alternate hypothesis that antenatal pelvic floor muscle exercise is effective in reducing the postnatal SUI is accepted. 
When comparing the incidence of SUI in both the experimental group and control group there was an incidence of $62 \%$ in the GE and $65 \%$ in control group. The severity of SUI experienced was higher in the control group when compared to the experimental group; the values being $06 \%$ and $55 \%$ between the scores I.01$2.0 .05 \%$ and $05 \%$ and $07 \%$ between the scores $2.01-3$ and $54 \%$ and $0 \%$ between the scores 3.01-4 respectively (Figure $1 \& 2$ ).
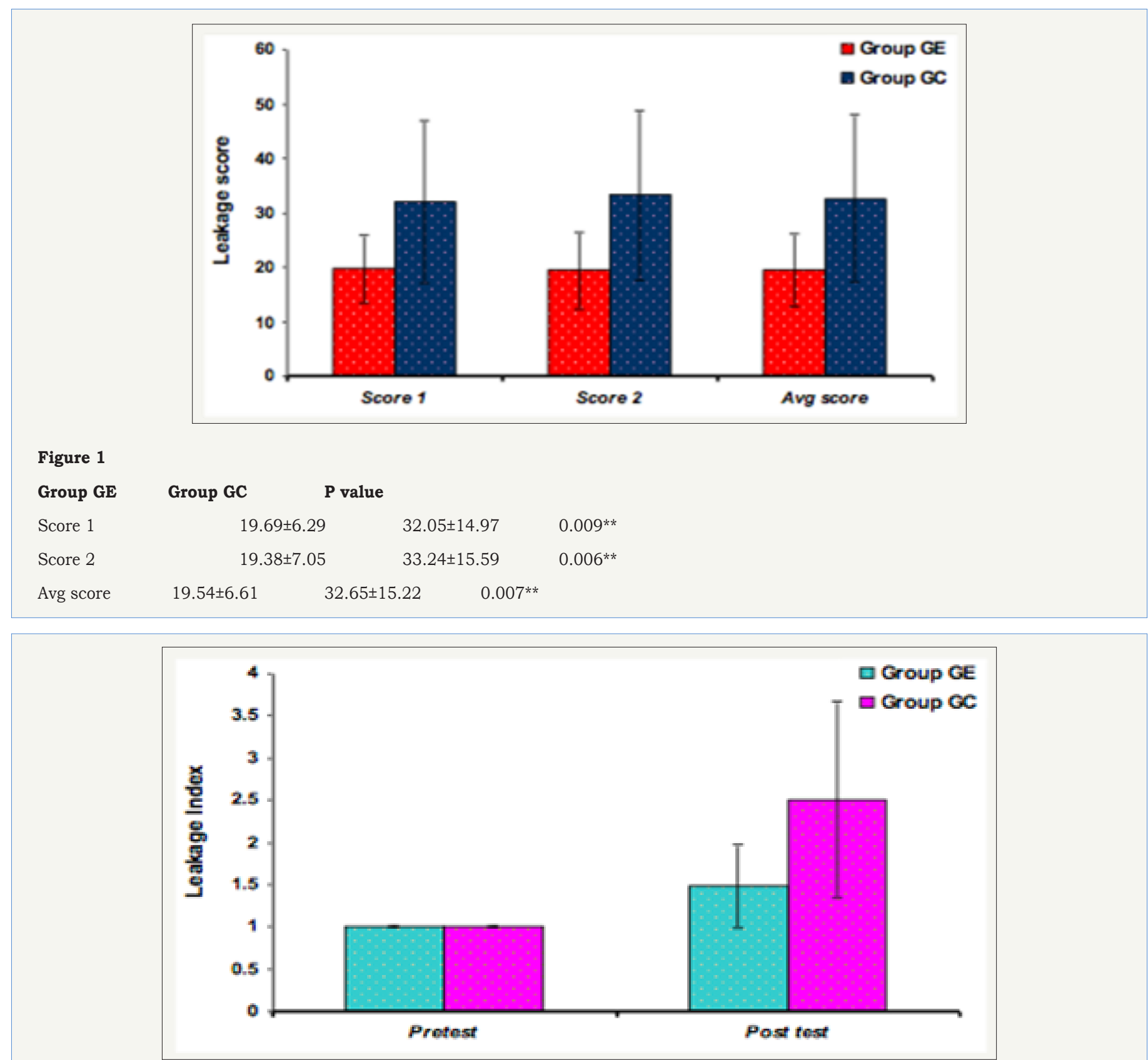

Figure 2: The comparison of Leakage Index in two groups studied.

$\begin{array}{lllc}\text { Group } & \text { GE Group } & \text { GCP value } & \\ \text { Pre test } & 1.00 & 1.00 & \text { NS } \\ \text { Post test } & 1.48 \pm 0.49 & 2.50 \pm 1.16 & 0.006^{* *} \\ \text { Difference } & 0.48 \pm 0.49 & 1.50 \pm 1.16 & 0.006^{* *}\end{array}$

\section{Discussion}

In this study 60 subjects were allocated at continence to the experimental (GE) and to the control group (GC). From the 6th month of pregnancy till term the subjects in experimental group was given Pelvic floor muscle training as at least a duration of 15 weeks is required to effectively train the muscle and any exercise 
in the first trimester is not recommended. The control group was given just the standard information and breathing exercise but not pelvic floor muscle training. The exercise session had both the fast and the slow Kegels exercise. The fast Kegels exercise was done 3 times daily as 5-10 rapid contractions. And for slow Kegels exercise the subjects were trained to contract their pelvic muscles for 10 seconds and then relax them for 10 seconds.

The subjects were asked to repeat the Kegel exercises 8 to 12 times each session, and do at least 3 sessions a day. The subjects in both the experimental and the control group who underwent a normal vaginal delivery were interviewed at the end of the $1 \mathrm{st}$ postpartum month (S1) and repeated after 2 weeks (S2) in regard to the SUI faced by them using the leakage index as the outcome measure. The average of both S1 and S2 was taken as S, this was done to minimize any errors that might have occurred like the chance of error due to postpartum heamorrhage ,so that the correctness of the data obtained can be maintained. The Leakage index (LI) was calculated using the formulae LI=average score(S)/ no of items on the scale [13].

There was a high rate of drop outs from the study owing to cesarean or assisted deliveries which are on a raise in India. The outcome measure used was leakage index which was put forward by Keri Bo. The scale had 13 physical activities which were to be marked from scores 1 to 5 . The total score was calculated and the leakage index was calculated was average score divided by 13 . The merits of the scale was that it would by carried out by the physiotherapist independently, unlike other outcome measures like the pad test which requires a gynaecologist to carry out the test. The reliability of the leakage index was found to be $r=.92$ by Keri Bo, hence better scale than the frequency diary method. Using the scale the level of severity of SUI experienced by the subjects was calculated. The demerits of the scale was that it doesn't give a score reference to classify the subject as continent, mild, moderate or severe incontinence, hence when the incidence of the incontinence was calculated those who had the leakage index less than 1 was considered to be continent and those above the leakage index of 1 was considered to be incontinent, whereas practically those subjects having a score between 1.1-2.0 experienced very little incontinence.

Other problem faced was that all the subjects were not ready to perform all the 13 items listed in the scale, eg jumping with subsequent abduction and adduction. Here the subjects were reluctant to perform the above said activity as they were uncomfortable or because of the fear. In such a scenario the total score obtained were have taken both the minimum value and the maximum value for the omitted item then using both the values the corresponding leakage index were found. There was no significant change in overall mean leakage index in both the cases ie any chance of biases was eliminated, in case the item was marked with the minimum value or the maximum value. The subjects were interviewed at the end of the 1 st postpartum month, and the total score S1 was obtained, the interview was repeated after 2 weeks and total score S2 was obtained.

The experimental group had a value of $19.69 \pm 6.29$ for S1 and that of S2 was $19.38 \pm 7.05$, here as seen there is a slight reduction in the values this might be attributed to the effect of the Kegels exercise and the better healing power of the trained muscles. This is supported by the studies of I Hahn who suggested that the healing power of the strength trained pelvic floor muscle is superior to that of the pelvic floor muscles that have not undergone any training [19]. Mean while the control group had a value of $32.05 \pm 14.97$ for S1 and $33.24 \pm 15.59$. for $\mathrm{S} 2$, here the values show an increase, the reason behind this is not fully understood, it might be due to the pattern of natural healing which have to be studied in detail. According to Guri Rortveit, Anne Kjersti Daltveit, Yngvild S, Hannestad, Steinar Hunskaar (2003).

The risk of urinary incontinence is higher among women who have had cesarean sections than among nulliparous women and is even higher among women who have had vaginal deliveries. Hence the mode of delivery was taken into consideration ie only those who underwent a normal vaginal delivery was considered for the study so as to avoid any OBG risk. Likewise the baby weight was also found and studied in relation to the postnatal SUI experienced by the mothers. All the babies were in the normal range of weight ie 2.5 to $3.5 \mathrm{~kg}$, hence any effect of the baby weight on the postnatal SUI were eliminated.

In the present study it was found that the antenatal pelvic floor muscle exercise is effective in reducing the severity of SUI experienced by the subjects who underwent the pelvic floor muscle training when compared to those who didn't undergo the pelvic floor muscle training exercise. This is in agreement with the findings of Hay Smith, Keri, Bo S Markved, Doumolin that the Kegels exercise is effective in reducing the SUI when done under the proper guidance of a physiotherapist. When comparing the post delivery leakage index in the experimental group and the control group with values in experimental group to be 1.0-2.61 and control group to be 1.0-3.73 with mean standard deviation of $1.48 \pm 0.49$ in experimental group and $2.50 \pm 1.16$ in the control group with $95 \%$ CI to be 1.18-1.79 and 1.90-3.09 in experimental group and control group respectively, the $\mathrm{P}$-value as found to be $\mathrm{P}=0.006$ and the effect size to be $1.06(\mathrm{~L})$. Hence in the present study antenatal PFMT was effective in reducing the severity of SUI experienced by the subjects who underwent the pelvic floor muscle training when compared to those who didn't undergo the pelvic floor muscle training exercise [20].

The presence of the therapist once a week did enhance the effects of the exercise and motivation of the subjects to continue the exercise throughout the following week. Also the guidance was useful to provide the corrections to the subjects in a properly individual tailored manner. When comparing the incidence of the SUI experienced by the two groups there was no much difference between the groups. The incidence rates were calculated using 
odds ratio and values were $62 \%$ in the experimental group and $68 \%$ in the control group. In the scale all those who had an LI of 1 is considered to be continent while those who have $>1$ was considered to be incontinent no matter how close the value was to 1. But the severity of SUI experienced by experimental group was significantly lesser when compared to that of the control group. The percentage of subjects who experienced SUI with a leakage index between 1.01-2 was 55\% in experimental group while it was $6 \%$ in the control group and those who experienced SUI with a leakage index between 3.01-4 in the experimental group was 0 while it was found to be $54 \%$ in the control group. From this data it is evident that the severity of SUI experienced by the experimental group was lesser than that experienced by those in control group. This shows that PFMT is effective in reducing SUI.

Hence it can be concluded that antenatal PFMT can be used to reduce the severity of SUI in postnatal period in primigravidae who underwent a normal vaginal delivery if not in reducing the incidence of postnatal SUI.

\section{Clinical Implications}

Results from this study suggest that antenatal pelvic floor muscle exercise is effective in reducing the severity of postnatal SUI if not decrease the incidence of postnatal SUI. Hence the result of the study may be helpful in giving awareness and education about antenatal pelvic floor muscle exercise and in general the antenatal care.

\section{Conclusion}

Antenatal pelvic floor muscle exercise is found to be effective in reducing the severity of postnatal SUI. When only the incidence is compared between two groups, the difference is not significant but the severity of SUI experienced by those who underwent the antenatal pelvic floor muscle exercise was lower when compared to those who did not undergo the antenatal pelvic floor muscle exercise.

\section{References}

1. Van der Kwaak A (1991) Women and health. Vena Journal 3(1): 2-33.

2. Sangeetha JMX (2010) A comparative study between comprehensive pelvic floor muscle training and conservative pelvic floor muscle training in prevention of stress urinary incontinence. The Indian Journal of Occupational Therapy XLII (1): 78-83.

3. Currie CJ, McEwan P, Poole CD (2006) The impact of the overactive bladder on the health related utility and quality of life. BJU 97: 12671272.

4. Bodhare TN, Valsangkar S, Bele SD (2010) An epidemiological study of urinary incontinence and its impact on quality of life among women aged 35 years and above in a rural area. Indian J Urol 26(3): 353-358.

5. Monz B, Pons ME, Hampel C, Hunskaar S, Quail D, et al. (2005) Patientreported impact of urinary incontinence-results from treatment seeking women in 14 European countries. Maturitas 52(2): 24-34.

6. Drutz HP, Herschorn S, Diamant NE (2005) Female pelvic medicine and reconstructive pelvic surgery. Springer publisher 11(16): 57-63.

7. Leanza V (2008) Pregancy and stress urinary incontinence (SUI): a literature review. Obstetrics \& Gynecology 78: 276-280.

8. Kumari S, Singh AJ, Jain V (2008) Treatment seeking behavior for urinary incontinence among north Indian women. Indian J Med Sci 62(9): 352356.

9. Thom DH, van den Eeden SK, Ragins AI, Wassel Fyr C, Vittinghof E, et al. (2006) Differences in prevalence of urinary incontinence by race/ ethnicity. J Urol 175(1): 259-264.

10. Th Dimpfl (1992) Incidence and cause of postpartum urinary stress incontinence. European Journal of Obstetrics \& Gynecology and Reproductive Biology 43(1): 29-33.

11. Minassian VA, Drutz HP, Al Badr A (2003) Urinary incontinence as a worldwide problem. Int J gynaecol obstst 82(3): 327-338.

12. Sharma JB, Aggarwal S, Singhal S, Kumar S, Roy KK (2009) Prevalence of urinary incontinence and other urological problems during pregnancy: a questionnaire based study. Arch Gynecol Obstet 279(6): 845-851.

13. Blaivas GJ, Groutz A (2002) Urinary incontinence: Epidemiology, pathophysiology, evaluation, and management overview. $\left(8^{\text {th }}\right.$ edn), WB Saunders, London, UK pp. 1207-1252.

14. Swanson JG, Kaczorowski J, Skelly J, Finkelstein M (2005) Urinary incontinence: Common problem among women over 45. Can Fam Physician 51: 84-85.

15. Bodhare, Sameer Valsangkar, Samir D, Bele (2010) An epidemiological study of urinary incontinence and its impact on quality of life among women aged 35 years and above in a rural area. Indian J Urol 26(3): 353358.

16. Fantl J (1996) Urinary incontinence in adults: acute and chronic management. Scand Journal Urology Nephrology 117: 681-682.

17. Judith Florendo (2008) Kegels De-Mystified for Women British Journal of Obstetrics \& Gynecology 26: 3-7.

18. K Bo (2007) Pelvic floor muscle exercise for treatment of stress urinary incontinence: An exercise physiology perspective. Journal International Urogynecology 6(5): 282-291.

19. Keri Bo (1999) Single blind, randomized controlled trial of pelvic floor exercises, electrical stimulation, vaginal cones, and no treatment in management of genuine stress incontinence in women. BMJ 318(7182): 487-493.

20. K Bo (1994) Reproducibility or instruments designed to measure subjective evaluation of female stress urinary incontinence. Scand J Urology and Nephrology 28(1): 97-100. 
(c) (i) Creative Commons Attribution 4.0 International License

For possible submissions Click Here

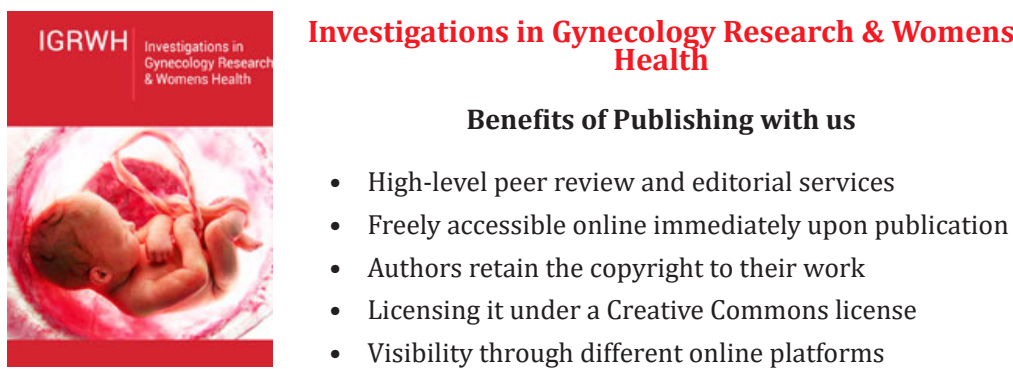

